\title{
Role of Genetic Polymorphism of Cyp2d6 in Tramadol Related Nephrotoxicity
}

\author{
Marwa Abass, Amal Elshal, Mohamad Hassan, Amina Elnaggar \\ Department of Forensic Medicine and Clinical Toxicology, Zagazig University, Zagazig, Egypt
}

Corresponding author: Marwa Abass, Department of Forensic Medicine and Clinical Toxicology, Faculty of Medicine, Zagazig University, Egypt, Tel: 20201279114301; Email: marwameriem@yahoo.com

Received date: August 10, 2016; Accepted date: August 30, 2016; Published date: September 06, 2016

Citation: Abass M. Role of Genetic Polymorphism of Cyp2d6 in Tramadol Related Nephrotoxicity. Med Toxicol Clin Forens Med. $2016,2: 2$.

Copyright: (c) 2016 Abass M. This is an open-access article distributed under the terms of the Creative Commons Attribution License, which permits unrestricted use, distribution, and reproduction in any medium, provided the original author and source are credited.

\section{Abstract}

Background: One of the most important tramadol metabolizing enzymes is CYP2D6. The CYP2D6 polymorphism leads to good, poor or no enzyme activity. Genetic testing helps in identification of high risk addicts whom to develop nephrotoxicity as well as management of addicts. The objective of this study is to explore tramadol induced nephrotoxicity associated with CYP2D6 gene polymorphism.

Methods: This study included 63 pure tramadol male addicts subdivided into ( 30 addicts' without nephrotoxicity and 33 cases with nephrotoxicity) and 78 healthy controls age and sex matched to the cases. The main major metabolite of tramadol mono-O-demethyl-tramadol (M1) was measured as tramadol exposure biomarkers. Biomarkers of nephrotoxicity including urinary neutrophil gelatinase-associated lipocalin (NGAL) levels were estimated using enzyme-linked immunosorbent assay (ELISA), and microalbumin values were measured by turbidimetric method. CYP2D6 gene polymorphism was detected by real time -polymerase chain reaction (RT-PCR).

Results: The duration of tramadol addiction was $12.7 \pm 5.2$ years with no significant association with nephrotoxicity. The CYP2D6 1/M and M/M genotypes distributions were significantly higher in tramadol addicts than controls. Moreover, the $\mathrm{M} / \mathrm{M}$ genotype or $\mathrm{M}$ allele was significantly associated with nephrotoxicity among tramadol addicts (OR (95\%Cl): 13.15 (1.48-111.37), $P=0.006$; OR (95\%Cl): 3.20 (1.46-7.02), $P=0.003$ respectively). There were significant increases of microalbuminuria and urinary NGAL; markers of renal toxicity, in homozygous $\mathrm{M} / \mathrm{M}$ and heterozygous $1 / \mathrm{M}$ genotypes followed by $1 / 1$ ( $P<0.001, P=0.001$, respectively). Logistic regression analysis revealed that tramadol addiction and CYP2D6 gene polymorphism were the main predictors of nephrotoxicity. Moreover, there was a significant decrease of tramadol metabolites levels among $M / M$ genotype.

Conclusion: CYP2D6 gene polymorphism is associated with susceptibility to tramadol induced nephrotoxicity. Larger scale studies are recommended to confirm previous results and genotyping testing is recommended for patients selection treated with tramadol.

\section{Keywords:}

CYP2D6 gene polymorphism; Tramadol addiction; Nephrotoxicity; Neutrophil gelatinase-associated lipocalin; Real time-polymerase chain reaction

\section{Introduction}

Tramadol is a powerful synthetic opioid analgesic agent, used for treatment of moderate to severe pain. The mechanism of its analgesic action is complex. Most reports suggest that the analgesic activity and other clinical effects of tramadol are a result of opioid and non-opioid mechanisms [1, 2]. In Egypt, numerous reports of abuse, dependence and overdose have been heavily demonstrated in the last few years as it is provided at cheap cost despite of being scheduled [3].

Tramadol biotransformation occurs in the liver. One of the most important tramadol metabolizing enzymes is CYP2D6. Metabolism results in o-desmethyl-tramadol, which itself are an active substance and 2 to 4 times more potent than tramadol [4]. The CYP2D6 polymorphism leads to good, poor or no enzyme activity. Genetic variant detection helps in identification of high risk addicts whom to develop toxicity as well as management of addicts [5].

CYP450 plays a pivotal role in the metabolism of multiple drugs and toxins [6]. One of the most important cytochrome P450 is CYP2D6 which metabolizes many drugs such as antidepressant and opiates including tramadol. In the liver tramadol undergoes extensive and complex metabolism via cytochrome P450 system, which converts it by o-demethylation into its active metabolite o-demethyltramadol (M1) [7]. M1 is the main metabolite of tramadol well known for its potent opioid activities through inhibition of reuptake of monoamines [8].

Several aspects of toxicokinetic properties of tramadol are not clearly understood especially the relationship between CYPP2D6 gene polymorphisms and tramadol toxicity. The highly polymorphic CYP2D6 plays an important role in determining the pharmacokinetics of tramadol and in predicting its adverse effects [9]. CYP2D6 genotype determines the level of the enzyme activity ('phenotype'). Individuals may carry either two 
functional wild-type alleles, or one functional wild-type allele. Different CYP2D6 genotypes may be zero (poor metabolizer), one (heterozygous individual ( $\mathrm{HZ}$ )/intermediate metabolizer), two (extensive metabolizer), and three active genes (ultra rapid metabolizer). The clinical effects of the CYP2D6 poor metabolizer genotype on pharmacokinetics of tramadol have been recorded in healthy volunteers and addicts. Tramadol abusers with ultra-rapid CYP2D6 metabolizers are at high risk for adverse effects or even intoxication [10].

Tramadol induced renal dysfunction is uncommon clinically [11]. Metabolites of drugs and toxicants that are excreted from kidneys may cause cellular damage leading to renal dysfunction. Urinary biomarkers such as microalbumin and neutrophil gelatinase-associated lipocalin (NGAL) proved clinically to be useful for assessment of functional integrity of glomeruli and proximal tubules [12].

Although the frequencies of CYP2D6 mutant alleles have been studied before among Egyptian population, but little is known about the relationship between CYP2D6 polymorphism and tramadol induced renal dysfunction [13]. Therefore, the aim of this study was to explore the possible association of tramadol addiction and its induced nephrotoxicity with CYP2D6 gene polymorphism.

\section{Materials and Method}

\section{Study population}

The current study was approved from the institutional review board, Zagazig Faculty of Medicine. This study included male tramadol addicts, ranging in age from 20 to 50 years, on voluntary bases, from those attending Psychiatry out-patient Clinic, Zagazig University Hospitals for treatment of drug addiction from May 2015 till June 2016. This pure tramadol addicts subdivided into (30 addicts without nepherotoxicity and 33 cases with nepherotoxicity) and 78 healthy controls age and sex matched to the cases. Participants were excluded from the study if they have history of kidney disease, urinary tract infection, heart disease, hypertension, diabetes mellitus, and cancer. Individual who had a previous or present exposure to agents capable of damaging the kidney (heavy metals such as lead, cadmium and other nephrotoxins such as organic solvents), regular and prolonged treatment by drugs affecting the kidney (e.g. aminoglycosides, antivirals...) were excluded from this study. All participants were subjected to interview using a questionnaire designed to obtain information on medical, occupational, family history and addiction symptoms.

\section{Sampling}

\section{Blood sampling}

Venous blood samples $(3 \mathrm{ml})$ were drawn from all subjects after an overnight fast and divided into two portions: $1 \mathrm{ml}$ of whole blood was collected into tubes containing ethylene diamine tetraacetic acid (EDTA) for detection of CYP2D6 gene polymorphism by TaqMan real-time polymerase chain reaction
(RT-PCR). Serum was separated immediately from remaining part of the sample and stored at $-20^{\circ} \mathrm{C}$ until analysis.

\section{Urine sampling and analysis}

A midstream urine sample will be collected by each participant into sterilized plastic container and centrifuged at $5000 \mathrm{rpm}$ for $5 \mathrm{~min}$ and then the clear supernatant was collected and stored for further analysis at $-20^{\circ} \mathrm{C}$.

The microalbuminuria as a sensitive one of kidney function tests was assessed. It is defined as an abnormal increase in albumin excretion rate/mg of creatinine in urine sample. Microalbuminuria was analyzed by TINA -QUANT on Cobas 6000 analyzer (Roche- Diagnostics, USA) as per description of Hofmann and Guder [14]. Urea was measured by urease GLDH method. Urinary and serum creatinine concentrations were assessed by kinetic Jaffie method using Cobas 6000 (Roche Diagnostics, USA) according to Tietz [15].

\section{Assessment of urinary major metabolite of tramadol}

The main major metabolite of tramadol: mono-o-demethyltramadol (M1) was measured in urine. Urine samples were assessed using Immune analysis EIA kit (Immunalysis Corporation, USA), DRI ${ }^{\circledR}$. This kit is a homogenous enzyme immunoassay kit using monoclonal antibodies which can detect mono-o-demethyl-tramadol (M1) the major main metabolite of tramadol in human urine.

\section{Measurement of urinary NGAL}

The neutrophil gelatinase-associated lipocalin levels (NGAL) in urine were measured using commercially available kit (MyBioSource, San Diego, CA, USA) according to the manufacturer's recommendations. NGAL is a small protein expressed in epithelia of renal tubules. A renal expression level of NGAL is dramatically increased after kidney injury within $2 \mathrm{~h}$ of the insult, making it an early and sensitive biomarker of kidney injury.

The assay is a sandwich enzyme-linked immune-sorbent assay (ELISA). The micro-wells were coated with a monoclonal antibody against human NGAL. Bound NGAL is detected with another monoclonal antibody labelled with biotin and the assay is developed with horseradish peroxidase (HRP)-conjugated streptavidin and a color-forming substrate. The color intensity is read at $450 \mathrm{~nm}$ in an ELISA reader. The color intensity (absorbance) is a function of the concentration of NGAL originally added to each well. The results for the calibrators are used to construct a calibration curve from which the concentrations of NGAL of test specimens are read.

\section{DNA extraction}

Genomic DNA was extracted from EDTA whole blood using a spin column method according to the protocol (QIAamp Blood Kit; Qiagen $\mathrm{GmbH}$, Hilden, Germany) DNA was stored at $-20^{\circ} \mathrm{C}$ till the time of use. 
Amplification of CYP2D6 gene polymorphism by TaqMan real-time PCR (RT-PCR)

The sequences of the primers and probes used in this study are listed in Table 1 . Primers and probe for CYP2D6 were designed using the Primer Express software, version 1.5 (Applied Biosystems, Foster City, CA). For CYP2D6 and albumin, primers and probes were used as described previously by Schaeffeler et al. and Aarskog and Vedeler [16, 17]. Primers were purchased from MWG (MWG-Biotech AG, Ebersberg, Germany), and probes were obtained from Applied Biosystems (Foster City, CA). Real-time PCR amplification was done using the ABI Prism 7700 sequence detection system. Amplification reactions ( $25 \mu \mathrm{l})$ were carried out in duplicate with 20 ng of template DNA, 1X TaqMan universal master mix buffer (Applied Biosystems, Foster City, CA), $300 \mathrm{nM}$ of each primer and $200 \mathrm{nM}$ of each fluorogenic probe. Thermal cycling was initiated with a 2 min incubation at $50^{\circ} \mathrm{C}$, followed by a first denaturation step of $10 \mathrm{~min}$ at $95^{\circ} \mathrm{C}$, and then by 40 cycles of $15 \mathrm{~s}$ at $95^{\circ} \mathrm{C}$ and of $1 \mathrm{~min}$ at $60^{\circ} \mathrm{C}$. In each assay, a standard curve was recorded and a no-DNA control was included. Relative quantification CYP2D6 amplification data were normalized to albumin as an internal reference gene using the 2- $\Delta \Delta \mathrm{Ct}$ method [18].

Table1 PCR primers and probes sequences for detection of CYP2D6gene polymorphism.

\begin{tabular}{|l|l|l|}
\hline Gene & Primers and probes sequences & PCR product size \\
\hline CYP2D6 & F:5'-CTTCACCTCCCTGCTGCAG -3' & 89 \\
\hline & R:5'-TCACCAGGAAAGCAAAGACA -3' & \\
\hline Albumin & Probe: 5'-FAM-CCGGCCCAGCCACCATGG-TAMRA & 72 bp \\
\hline & F:5'-TGTTGCATGAGAAAACGCCA-3' & \\
\hline & R:5'-GTCGCCTGTTCAACCAAGGAT -3' & Probe: 5'-FAM-AAGTGACAGAGTCACCAAATGCTGCACAG-TAMRA \\
\hline
\end{tabular}

\section{Statistical analysis}

Statistical analyses were performed using the Statistical Package for the Social Sciences for Windows (version 17.0; SPSS Inc., Chicago, IL, USA). The results for continuous variables were expressed using descriptive statistic (mean \pm standard deviation) and were analyzed using " $\mathrm{t}$ " test. Allelic and genotypic frequencies were compared and statistically analyzed using the Fisher's exact test and Odds ratio (OR) with 95\% confidence intervals $(95 \% \mathrm{Cl})$. Conformity of genotype distributions with Hardy-Weinberg (HW) equilibrium was evaluated by Chi-square analysis. One way analysis of variance (ANOVA) test was done to compare different parameters between more than two groups. Logistic regression analysis was used to evaluate the association between two continuous variables. For all tests, a P-value $<0.05$ was considered to be statistically significant.

\section{Results}

Clinical and biochemical characteristics' of the studied group

The duration of tramadol addiction was $12.7 \pm 5.2$ years with no significant association with nephrotoxicity. There was significantly higher microalbuminuria and urinary NGAL levels in pure tramadol addicts as compared to healthy controls $(P<0.001$ for each), while we didn't find any significant differences between two groups as regard age, serum creatinine and urea (Figure 1).

\section{CYP2D6 gene polymorphism among pure tramadol addicts and healthy controls}

Genotype and allelic frequencies of CYP2D6 gene polymorphism in tramadol addicts and healthy volunteers were presented in Table 2. The genotypes distributions were in HardyWeinberg equilibrium in each studied group.

The wild (1/1) genotype of CYP2D6 gene was prevailed in two groups (healthy controls (71.8\%); and in pure tramadol addicts (48.4\%). The minor allele frequency (MAF), M allele, was $16.7 \%$ in controls and $33.3 \%$ of tramadol addicts. The $1 / \mathrm{M}$ and $\mathrm{M} / \mathrm{M}$ genotypes distributions were significantly higher in tramadol addicts than controls (1/M: $36.4 \%$ vs. $23.1 \%$; $M / M$ : $15.2 \%$ vs. $5.1 \%)$. Risk of tramadol addiction was significantly higher among patients carrying $1 / \mathrm{M}$ and $\mathrm{M} / \mathrm{M}$ genotypes than those carrying 1/1 genotype (OR $(95 \% \mathrm{Cl}): 2.3(1.10-4.94), P=0.003$; OR $(95 \%$ $\mathrm{Cl})$ : 4.4(1.27-15.09), $\mathrm{P}=0.01$, respectively) or at least carrying one $\mathrm{M}$ allele than among patients carrying the wild allele 1 (OR $(95 \%$ Cl): 2.5 (1.43-4.36), $P=0.001)$.

Table2 CYP2D6 genotypes and alleles frequencies in pure tramadol addict's and healthy controls, 1=wild allele; M=mutant alleles of CYP2D6, i.e., alleles 3, 4 and 5; OR: odds ratio; $95 \% \mathrm{Cl}$ : $95 \%$ confidence interval, ${ }^{*} \mathrm{P}$ significant $<0.05$ compared with controls.

\begin{tabular}{|c|c|c|c|c|}
\hline CYP2D6 gene & Healthy controls & Pure tramadol addicts & OR (95\% Cl) & P \\
\hline & $\mathrm{n}=\mathbf{7 8}$ & $\mathrm{n}=66$ & \\
\hline
\end{tabular}




\begin{tabular}{|l|c|c|c|c|c|}
\hline & $\mathbf{n}$ & $\mathbf{( \% )}$ & $\mathbf{n}$ & $\mathbf{( \% )}$ & \\
\hline $1 / 1$ & 56 & -71.8 & 32 & -48.4 & \\
\hline $1 / \mathrm{M}$ & 18 & -23.1 & 24 & -36.4 & $2.3(1.10-4.94)$ \\
\hline M/M 4/4 & 4 & -5.1 & 10 & -15.2 & $4.4(1.27-15.09)$ \\
\hline Allele 1 & 130 & -83.3 & 88 & -66.7 & $0.03^{*}$ \\
\hline Allele M & 26 & -16.7 & 44 & -33.3 & $2.5(1.43-4.36)$ \\
\hline
\end{tabular}

CYP2D6 gene polymorphism among pure tramadol addicts with or without nephrotoxicity

We further analyzed our data by stratifying tramadol addicts according to the presence or absence of nephrotoxicity. The tramadol addicts had higher frequencies of CYP2D6 M/M genotype ( $25 \%$ vs. $3.3 \%$ ) and M allele ( $44.4 \%$ vs. $20 \%$ ), while the heterozygous ( $M / 1)$ genotype was not significantly different between two groups. It was evident that the presence of the $\mathrm{M} / \mathrm{M}$ genotype or $\mathrm{M}$ allele was significantly associated with nephrotoxicity among tramadol addicts (OR $(95 \% \mathrm{Cl}): 13.15$ (1.48-116.73), $\mathrm{P}=0.006$; OR (95\% Cl): 3.20 (1.46-7.02), $\mathrm{P}=0.003$, respectively) (Table 3 ).

Table 3 CYP2D6 genotypes and alleles frequencies in pure tramadol addict's without or with nephrotoxicity, 1=wild allele; M=mutant alleles of CYP2D6 i.e., alleles 3, 4 and 5; OR: odds ratio; $95 \% \mathrm{Cl}$ : 95\% confidence interval, ${ }^{*} \mathrm{P}$ significant $<0.05$ compared with tramadol addicts cases without nephrotoxicity.

\begin{tabular}{|c|c|c|c|c|c|c|}
\hline \multirow[t]{3}{*}{ CYP2D6 gene } & \multicolumn{2}{|c|}{ Tramadol addicts without nephrotoxicity } & \multicolumn{2}{|c|}{ Tramadol addicts with nephrotoxicity } & OR $(95 \% \mathrm{CI})$ & $\mathrm{P}$ \\
\hline & \multicolumn{2}{|r|}{$n=30$} & \multicolumn{2}{|r|}{$n=36$} & & \\
\hline & $\mathbf{n}$ & $(\%)$ & $\mathbf{n}$ & $(\%)$ & & \\
\hline $1-1$ & 19 & -63.4 & 13 & -36.1 & & \\
\hline $1 / \mathrm{M}$ & 10 & -33.3 & 14 & -38.9 & $2.05(0.70-5.99)$ & 0.19 \\
\hline $\mathrm{M} / \mathrm{M} 4 / 4$ & 1 & -3.3 & 9 & -25 & $13.15(1.48-116.73)$ & $0.006^{*}$ \\
\hline Allele 1 & 48 & -80 & 40 & -55.6 & & \\
\hline Allele M & 12 & -20 & 32 & -44.4 & $3.20(1.46-7.02)$ & $0.003^{*}$ \\
\hline
\end{tabular}

Clinical and biochemical parameters in tramadol addicts among different CYP2D6 genotypes

We further analyzed Clinical and biochemical parameters in tramadol addicts among different CYP2D6 genotypes to assess the effect of each genotype on these parameters. There was a significant increase in microalbuminuria and urinary NGAL and a significant decrease in tramadol's metabolite level, o-demethyl tramadol (M1) in homozygous $\mathrm{M} / \mathrm{M}$ and heterozygous $1 / \mathrm{M}$ genotypes followed by $1 / 1 \quad(P<0.001, P=0.001, P<0.001$, respectively). However, we didn't find any significant differences in other parameters between three genotypes among tramadol addicts (Table 4).

Table 4 Clinical and biochemical parameters in tramadol addict's among different CYP2D6 genotypes, ${ }^{*}$ Significant difference with in CYP2D6 among tramadol addicts.

\begin{tabular}{|c|c|c|c|c|}
\hline \multirow{4}{*}{ Parameter } & \multicolumn{3}{|c|}{ Tramadol addicts } & \multirow{2}{*}{$\mathrm{F}$} \\
\hline & \multicolumn{3}{|c|}{$n=66$} & \\
\hline & $1-1$ & $1 / M$ & $M / M$ & \\
\hline & $n=32$ & $n=24$ & $n=10$ & \\
\hline Age (years) & $31.1 \pm 6.7$ & $33.4 \pm 7.1$ & $32.6 \pm 7.9$ & 0.21 \\
\hline Duration of tramadol addiction & $11.5 \pm 5.9$ & $12.6 \pm 4.8$ & $10.8 \pm 6.9$ & 0.45 \\
\hline Serum creatinine (mg/dl) & $0.71 \pm 0.24$ & $0.75 \pm 0.25$ & $0.76 \pm 0.28$ & 0.61 \\
\hline Serum urea (mg/dl) & $11.4 \pm 1.8$ & $11.9 \pm 1.6$ & $12.1 \pm 1.1$ & 0.15 \\
\hline
\end{tabular}




\begin{tabular}{|l|c|c|c|}
\hline Micro albuminuria/U.cret. ( $\mathrm{gg} / \mathrm{mg}$ creatinine) & $49.7 \pm 13.9$ & $64.9 \pm 17.8$ & $75.4 \pm 19.1$ \\
\hline Urine NGAL $(\mathrm{pg} / \mathrm{mL})$ & $29.7 \pm 10.2$ & $30.2 \pm 6.1$ & $38.4 \pm 5.3$ \\
\hline Mono-o-demethyl-tramadol $(\mathrm{M} 1)(\mathrm{ng} / \mathrm{ml})$ & $381 \pm 8.5$ & $241 \pm 10.5$ & $101 \pm 6.5$ \\
\hline
\end{tabular}

Logistic regression analysis for prediction of nephrotoxicity in tramadol addicts and healthy controls

Univariate logistic regression analyses were performed to clarify the main predictors' nepherotoxicity. CYP2D6 gene polymorphism and tramadol addiction were selected as significant predictors associated with the nephrotoxicity. Our findings revealed that tramadol addiction and CYP2D6 gene polymorphism were significantly predictors of nephrotoxicity (odds ratio: $2.358, \mathrm{P}<0.001$; odds ratio: $1.987, \mathrm{P}=0.001$, respectively) (Table 5).

Table 5 Logistic regression analysis to predict of nephrotoxicity in tramadol addicts and healthy controls.

\begin{tabular}{|c|c|c|c|c|c|c|c|}
\hline & \multicolumn{2}{|c|}{ Unstandardized coefficients } & \multirow{2}{*}{$\begin{array}{l}\begin{array}{l}\text { Standardized } \\
\text { Coefficients }\end{array} \\
\beta\end{array}$} & \multirow{2}{*}{$\begin{array}{l}\text { 95\% C.I. } \\
\text { Lower Bound }\end{array}$} & \multirow[b]{2}{*}{ Upper Bound } & \multirow[t]{2}{*}{$\mathbf{t}$} & \multirow[t]{2}{*}{$P$ value } \\
\hline & $\beta$ & Standard error & & & & & \\
\hline Constant & 6.45 & 1.09 & & & & 4.73 & $<0.001$ \\
\hline Tramadol addiction & 0.92 & 0.18 & 2.358 & 1.47 & 3.79 & 3.67 & $<0.001$ \\
\hline CYP2D6 gene polymorphism & 1.03 & 0.23 & 1.987 & 1.28 & 2.98 & 2.98 & 0.001 \\
\hline
\end{tabular}

\section{Discussion}

Pharmacokinetic studies have shown that CYP2D6 plays an important role of in tramadol metabolism. The variability of tramadol pharmacokinetic properties has been related partly to CYP2D6 polymorphism making it a current topic of investigation to understand the inter-individual difference to help clinicians to individualize the drug treatment by selecting appropriate therapies [19].

Many renal diseases are associated with substance of abuse by varied mechanisms. The majority of these substances, or their metabolites, are excreted via the kidney. Some substances may be directly or indirectly nephrotoxic. A number of mechanisms are involved such as rhabdomyolysis, atherosclerosis, hypotension, hypoxia and acidosis [20].

Data of the present study showed a significant increase in urinary microalbuminuria and NGAL $(P<0.001)$ among tramadol addicts compared to healthy controls. These results indicated that structural and functional integrity of both glomeruli and proximal tubules are deteriorated with tramadol addiction (Figure 1).
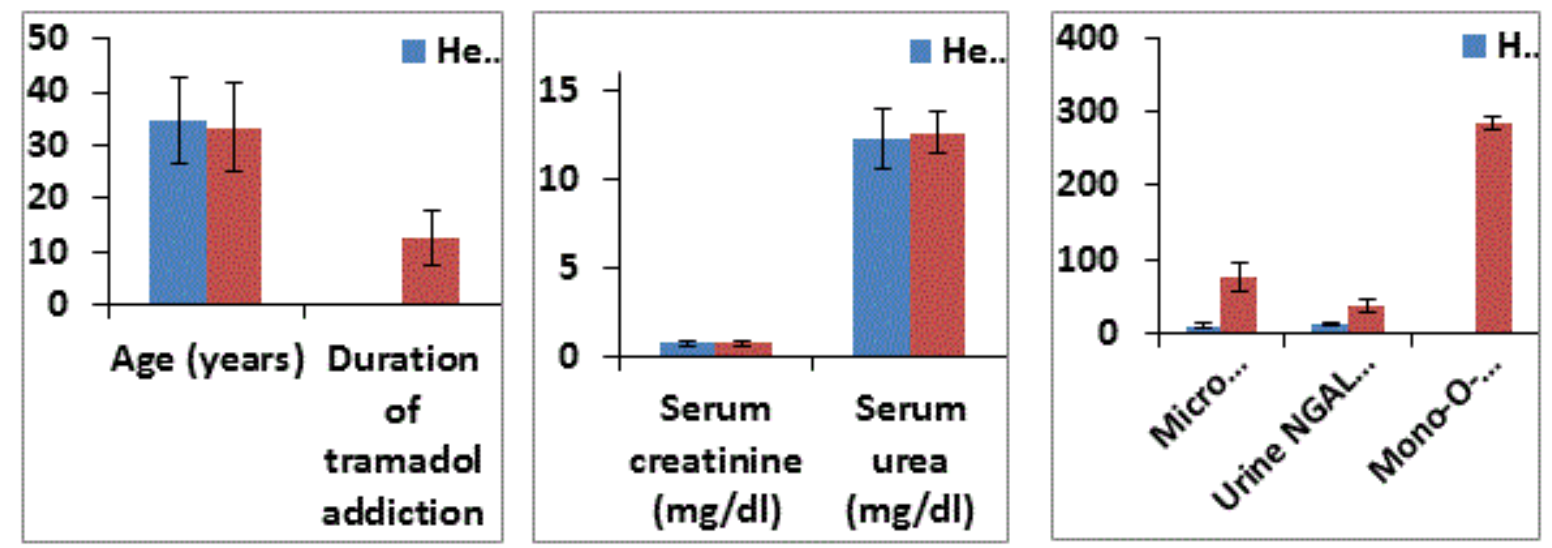

Figure 1 Clinical and biochemical characteristics of the studied groups.

Using urine samples for detection and quantitation of tramadol metabolites has many advantages such as being noninvasive and large volumes and large number of samples can be easily collected [21].
This study highlights the CYP2D6 allelic variants' distribution among Egyptian tramadol addicts. $66.7 \%$ of the tramadol addicts had a wild type of CYP2D6 allelic variants $\left({ }^{*} 1\right)$ and the most common inactive allelic variants were $\left({ }^{*} 4\right) .60 \%$ of tramadol addicts had a wild type of CYP2D6 allelic variants $\left({ }^{*} 1\right)$ and the 
most common mutant allelic variants were $\left({ }^{*} 4\right)$. Comparing cases of tramadol addicts with nephrotoxicity and addicts without nephrotoxicity as regard to the genotypic allelic variants of CYP2D6 gene, nephrotoxicity cases showed a significantly lower wild genotype $1 / 1$ compared to addicts without nephrotoxicity $(36.1 \%$ vs. $63.4 \%, p<0.001)$ with a higher frequency of mutant genotype $4 / 4$ ( $25 \%$ vs. $3.3 \%, p=0.006)$. We are in the same line with Ibrahim et al. who studied CYP2D6 allelic variants among the Egyptian tramadol intoxicated patients [13]. Moreover, the highest concentration of mono-o-demethyltramadol (M1) was recorded in homozygous 1/1and heterozygous $1 / \mathrm{M}$ genotypes followed by $M / M$. In accordance with our results Halling et al. who found that individuals with mutant CYP2D6 alleles (e.g. ${ }^{*} 3,{ }^{*} 4,{ }^{*} 4 x n,{ }^{*} 10,{ }^{*} 17$ or $^{*} 5$ ) had minimal or absent enzymatic activity with higher tramadol concentration [4]. Albumin present in the urine is often the first indicator of glomerular damage, and a decline in renal function [22]. Results of the present study for the effect of tramadol addiction on glomerular function showed significant decrease in microalbuminurea in tramadol addicts as compared to healthy control, suggesting glomerular damage. This result is accordance with finding of other investigators who reported that multiple histological changes in the form of atrophied glomeruli with collapsed tufts, wide Bowman's space, degenerated tubules and cellular infiltrations were observed in experimental rats given tramadol chronically for 30 days [23, 24].

Recently NGAL is considered to be one of the earliest and most robustly induced proteins in the kidney after ischemia or nephrotoxicity in animal models. Importantly, NGAL protein is easily detected in the blood and urine soon after renal damage in pre-clinical studies [25]. These findings have initiated a number of translational studies to evaluate NGAL as a novel biomarker in human renal disease. In the current study tramadol addiction affected proximal tubular function showed an increase in the urinary excretion of NGAL, suggesting impairment in renal proximal tubular function, and tubulointerstitial nephropathy. This suggestion is supported by other investigators who reported histopathological changes in renal tubules due to tramadol chronic toxicity in animal experiments, and in human post-mortem microscopy examination of a young patient who died of fatal tramadol overdose due to acute tubular necrosis of the kidney $[23,26,27]$.

\section{Conclusion}

CYP2D6 polymorphism is positively affecting the tramadol induced renal dysfunction in tramadol addicts. This study represented that CYP2D6*1 (wild type) was the most common variant among Egyptian population and $\mathrm{CYP}^{2} \mathrm{D} 6^{*} \mathrm{M}$, mutant allele, was predominant in tramadol addicts with renal dysfunction. We found a significant higher concentration of tramadol metabolite (M1) level in CYP2D6*1 allelic variants compared to other variant, which clearly pointed to the tramadol induced renal toxicity is largely induced by tramadol with minimal role of $\mathrm{M} 1$. The current study results are restricted by some limitations such as the relatively small number of studied patients, especially in subgroup analysis, so further multicenter studies are warranted involving larger sample of tramadol addicts to clarify tramadol toxico kinetics in each specific phenotype and search for other important interactive genomic markers.

\section{Acknowledgement}

We are indebted to all contributors in this paper.

\section{Financial Disclosure}

The authors declare that this work was not funded by any sponsor.

\section{Conflict of Interest}

The authors declare that they have no conflicts of interest. 


\section{References}

1. Grond S, Sablotzki A (2004) Clinical pharmacology of tramadol. Clin Pharmacokinet 43: 879-923.

2. Gillman PK (2005) Monoamine oxidase inhibitors, opioid analgesics and serotonin toxicity. Br J Anaesth 95: 434-441.

3. Al Akabawi A (2001) Drug abuse in the Arab world. A country profile of Egypt. In Images in Psychiatry. Arab Perspective, World Psychiatric Association 143-150.

4. Halling J, Weihe P, Brosen K (2008) CYP2D6 polymorphism in relation to tramadol metabolism: A study of faroese patients. Ther Drug Monit 30: 271-275.

5. Bressolle F, Rochette A, Khier S, Dadure C, Ouaki J, et al. (2009) Population pharmacokinetics of the two enantiomers of tramadol and O-demethyl tramadol after surgery in children. $\mathrm{Br} \mathrm{J}$ Anaesth 102: 390-399.

6. Zhou SF (2009) Polymorphism of human cytochrome P450 2D6 and its clinical significance: Part II. Clin Pharmacokinet 48: 761-804.

7. Pedersen RS, Damkier P, Brosen K (2005) Tramadol as a new probe for cytochrome P450 2D6 phenotyping: A population study. Clin Pharmacol Ther 77: 458-467.

8. Arjunan V, Santhanam R, Marchewka MK, Mohan S (2014) Comprehensive quantum chemical and spectroscopic (FTIR, FTRaman, $1 \mathrm{H}, 13 \mathrm{C}$ NMR) investigations of O-desmethyltramadol hydrochloride an active metabolite in tramadol-An analgesic drug. Spectrochim Acta A Mol Biomol Spectrosc 122: 315-330.

9. Gan SH, Ismail R, Wan Adnan WA, Zulmi W (2007) Impact of CYP2D6 genetic polymorphism on tramadol pharmacokinetics and pharmacodynamics. Mol Diagn Ther 11: 171-181.

10. Stamer UM, Musshoff F, Kobilay M, Madea B, Hoeft A, et al. (2007) Concentrations of tramadol and O-desmethyltramadol enantiomers in different CYP2D6 genotypes. Clin Pharmacol Ther 82: 41-47.

11. Wang SQ, Li CS, Song YG (2009) Multiply organ dysfunction syndrome due to tramadol intoxication alone. Am J Emerg Med 27: 903.

12. Koyner JL, Garg AX, Coca SG, Sint K, Thiessen-Philbrook H, et al. (2012) Biomarkers predict progression of acute kidney injury after cardiac surgery. J Am Soc Nephrol 23: 905-914.

13. Ibrahim SF, Ali MM, Gouda AS, Rashed LA (2015) Association between the severity of tramadol toxicity and some CYP2D6 allelic variants in Egyptian tramadol intoxicated patients. Emergency Med 6: 303.

14. Hofmann W, Guder WG (1989) A diagnostic programme for quantitative analysis of proteinuria. J Clin Chem Clin Biochem 27: 589-600.
15. Tietz NW (1976) Textbook of Clinical Chemistry, Philadelphia W.B Saunders

16. Schaeffeler E, Schwab M, Eichelbaum M, Zanger UM (2003) CYP2D6 genotyping strategy based on gene copy number determination by TaqMan real-time PCR. Hum Mutat 22: 476-485.

17. Aarskog NK, Vedeler CA (2000) Real-time quantitative polymerase chain reaction. A new method that detects both the peripheral myelin protein 22 duplication in Charcot-Marie- Tooth type $1 \mathrm{~A}$ disease and the peripheral myelin protein 22 deletion in hereditary neuropathy with liability to pressure palsies. Hum Genet 107: 494-498.

18. Livak KJ, Schmittgen TD (2001) Analysis of relative gene expression data using real-time quantitative PCR and the 2(-delta delta $C(T)$ ) method. Methods 25:402-408.

19. Ardakani YH, Rouini MR (2007) Pharmacokinetics of tramadol and its three main metabolites in healthy male and female volunteers. Biopharm Drug Dispos 28: 527-534.

20. Crowe AV, Howse M, Bell GM, Henry JA (2000) Substance abuse and the kidney QJM 93: 147-152.

21. Ismaiel OA, Hosny MM (2012) Development and validation of a spectrophotometric method for the determination of tramadol in human urine using liquid-liquid extraction and ion pair formation. Int J Inst Sci 1: 34-40.

22. El-Safty IAM, Mohy El-Deen IE, El-Rasheed AH, El-Ashmawy MFM (2015) Renal effects of tramadol addiction and cannabinoid abuse. RJPBCS 6: 1212-1218

23. Atici S, Cinel I, Cinel L, Doruk N, Eskandari G, et al. (2005) Liver and kidney toxicity in chronic use of opioids: an experimental long term treatment model. J Biosci 30: 245-252.

24. Elkhateeb A, El Khishin I, Megahed O, Mazen F (2015) Effect of Nigella sativa Linn oil on tramadol-induced hepato- and nephrotoxicity in adult male albino rats. Toxicology Reports 39: 512-519.

25. Devarajan $P$ (2008) Neutrophil gelatinase-associated lipocalin (NGAL): A new marker of kidney disease. Scandinavian Journal of Clinical and Laboratory Investigation Supplementum 241: 89-94.

26. De Decker K, Cordonnier J, Jacobs W, Coucke V, Schepens P, et al. (2008) Fatal intoxication due to tramadol alone: Case report and review of the literature. Forensic Sci Int 175: 79-82.

27. Ali AA, Wassim NM, Dowaidar MM, Yaseen AE (2013) Genetic polymorphism of CYP2D6 gene among Egyptian hypertensive cases. The Journal of Basic \& Applied Zoology 66: 228-233. 